\title{
Editorial
}

\section{Stem Cell in Spinal Cord Injury: Hype or A New hope?}

Spinal cord injury (SCI) occurs most commonly in young adults due to high-velocity trauma and fall from the height, leaving behind the lifelong physical, psychological and social disability. There is no effective medical or surgical treatment yet available to reverse the disability produced in sequence to injury. Cavitation and loss of neural and stromal tissue at the time of SCI result in not only sensory-motor loss but also autonomic dysfunction. Residual functional cord tissue is not capable of reconstituting the gap already created in the spinal cord; on the other hand remodeling results in glial scar formation but no neural tissue is formed. Among the treatment options available and explored in recent research, use of stem cells is capable of bridging the area of cavitation and neuronal regeneration in glial scar tissue as shown in animal and human studies. ${ }^{1}$ Hence, stem cell therapy is considered to be a promising treatment option in SCI, though in its evolutionary phase.

Till date, no trial has shown a level of effectiveness that gives hope regarding its potential to be useful as mainline therapy nor any conscience or guidelines regarding stem cells usage has been laid and practiced in spirit.

The government of India lends generous financial and infrastructural support to various government institutes, private sector undertakings, and industries to conduct stem cell research, which would help to decrease the load of disability and improve the cost of living and functional status of patients suffering from neurological disorders. ${ }^{2}$ Phase I trials have been carried out in India but without any promising results.

Because of the unique characteristic of stem cells, it's being used as potential treatment options for spinal cord injury, traumatic brain injury, and degenerative brain diseases. Considering characteristic potential and abilities of self-renewal and differentiation into any cell lineage in the body, use of stem cells in degenerative conditions for regeneration is justified.

The Department of Orthopaedics in Postgraduate Institute of Medical Education and Research did a couple of studies using mesenchymal stem cells and olfactory nerve sheath cells in chronic SCI but is way away from reporting any remarkable and notifiable success along with recommending its usage in such scenarios and circumstances.

Due to the complex pathology of SCI, developing an effective curative treatment in clinical practice is difficult. Most of the current modalities are directed and aimed to the reversal of the damaged cells. Researchers used several molecular therapies for SCI for a better outcome of injury. Use of stem cell therapy results in regeneration of axons from precursor oligodendrocytes by virtue of its growth factor inducing anti-inflammatory property. Of the various sources of stem cells, bone marrow derived mesenchymal stem cells are used widely in research programs due to ethical issues associated with stem cell procurement.

Although FDA approved (2009) the use of stem cell therapy in humans but faced criticism due to limited human information as most of the animal study was done on rodents but not on the larger animals similar to human being. At present stem cell therapy in SCI with the multicentric human study are on progress with promising results. Stem cells and regenerative medicine bring new hope to persons with SCI, but practitioners and researchers should be mindful of the complex ethical issues involved with this novel treatment. Difficult questions are being asked about the ethical validity of creating new embryos purely for the purpose of research, as well as new cellular techniques, the creation of chimeras, parthenogenetic activation, and cytoplasmic transfer. Such issues are raised in the context of a global bio-health economy that is more often seeing the cellular material as intellectual property, capable of being the subject of trade. ${ }^{3}$

\section{REFERENCES}

1. Oh SK, Jeon SR. Current Concept of Stem Cell Therapy for Spinal Cord Injury: A Review. Korean J Neurotrauma. 2016;12(2):40-46.

2. Bhasin A, Padma Srivastava MV, Bhatia R, Kumaran S, Mohanty S (2014) Stem Cells in Neurological Diseases: Indian Perspective. J Stem Cell Res Ther. 2014;4:175.

3. Jeffrey V. Rosenfeld, Prateek Bandopadhayay, Tony Goldschlager, Douglas J. Brown.The Ethics of the Treatment of Spinal Cord Injury: Stem Cell Transplants, Motor Neuroprosthetics, and Social Equity.Top Spinal Cord Inj Rehabil. 2008 Summer; 14(1):76-88.

Vishal Kumar Assistant Professor Department of Orthopaedics Postgraduate Institute of Medical Education and Research Chandigarh, India

Sarvdeep Singh Dhatt Associate Professor Department of Orthopaedics

Postgraduate Institute of Medical Education and Research Chandigarh, India 